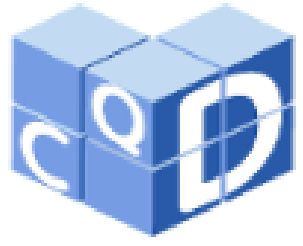

Revista Eletrônica

Paulista de Matemática

ISSN 2316-9664

Volume 16, dez. 2019

Rogério César dos Santos

FUP/UnB

rogerc@unb.br

Breno Ramires Vargas da
Silva
SEE/DF
brenoramires23@hotmail.com
Carlos Derli Almeida
Cornélio
UEG-Formosa
matcarlosderli@gmail.com

Letícia dos Anjos Rodrigues SEE-DF

leticiaanjosr@gmail.com

\section{Extensões do Teorema de Van Aubel para octógonos}

\author{
Van Aubel Theorem extensions for octagons
}

\begin{abstract}
Resumo
Demonstraremos neste artigo uma propriedade dos octógonos que amplia o resultado obtido por Silva (2018), e que guarda semelhanças com o conhecido Teorema de Van Aubel, da Geometria Plana. Números complexos e vetores são as ferramentas usadas na prova. A conclusão que chegamos é que padrões podem surgir quando usamos pontos centrais de objetos matemáticos em situações que envolvem figuras geométricas construídas sobre outras.

Palavras-chave: Octógonos, Van Aubel.
\end{abstract}

\begin{abstract}
We will demonstrate in this article a property of octagons that broadens the result obtained by Silva (2018) and which bears similarities with the well-known Van Aubel Theorem of Classic Geometry. Complex numbers and vectors will be the tools used in the proofs. The conclusion we have come to is that patterns can arise when we use center points of mathematical objects in situations involving geometric figures built over others.
\end{abstract}

Keywords: Octagons, Van Aubel. 


\section{Introdução}

O Teorema de Van Aubel (NISHIYAMA, 2011) diz que, dado um quadrilátero qualquer $A B C D$, então, se quadrados são construídos externamente sobre seus lados, os dois segmentos $M O$ e $N P$, que unem os pares de centros dos quadrados opostos, são ortogonais e congruentes, como ilustra a figura 1.

O referido teorema constitui-se em fonte inesgotável de inspiração para a descoberta de novos padrões que podem ser obtidos por construções geométricas que envolvem pontos médios de segmentos e centros de polígonos, como podemos ver em trabalhos como o de Krishna (2018) e Silva (2018), por exemplo.

Inspirados em construções semelhantes, iremos demonstrar neste artigo uma proposição relativa aos octógonos, que julgamos ser inédita.

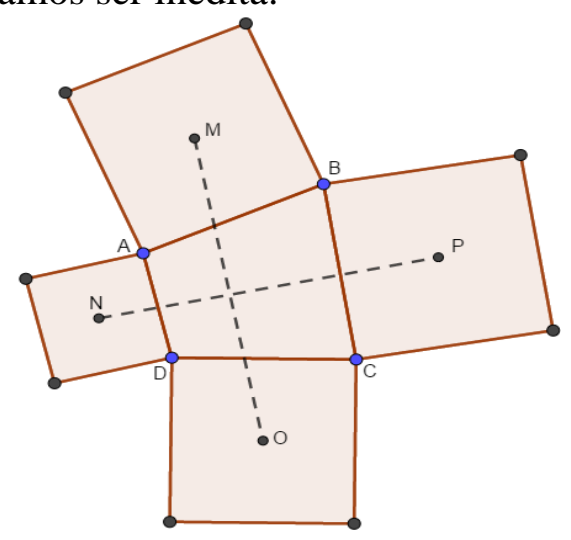

Figura 1 - Teorema de Van Aubel: segmentos $M O=N P, M O \perp N P$

Fonte: construção no software GeoGebra.

É útil comentarmos, neste momento, a prova do teorema de Van Aubel acima descrito, pois as demonstrações dos resultados que vamos apresentar neste artigo contém ideias semelhantes às do mesmo. Em linhas gerais, a prova de Van Aubel consiste em, inicialmente, considerar, na figura 1, que cada vértice do quadrilátero $A B C D$ é um ponto do plano cartesiano, de duas coordenadas. Logo, podemos associar a cada vértice de $A B C D$ ao seu número complexo correspondente, assim: o ponto $A=\left(a_{1}, a_{2}\right)$ está associado ao complexo $a^{\prime}=a_{1}+a_{2} i$, o ponto $B=\left(b_{1}, b_{2}\right)$ está associado ao complexo $b^{\prime}=b_{1}+b_{2} i$, e assim sucessivamente, até o ponto $D$. Logo, $a^{\prime}, b^{\prime}, c^{\prime}$ e $d^{\prime}$ são os números complexos associados aos vértices do quadrilátero $A B C D$, nesta ordem.

Em seguida, tomam-se as diferenças $z_{1}=a^{\prime}-d^{\prime}, z_{2}=b^{\prime}-a^{\prime}, z_{3}=c^{\prime}-b^{\prime}$ e $z_{4}=d^{\prime}-$ $c^{\prime}$. Agora, considerando $m, n, o$ e $p$ os números complexos associados aos pontos $M, N, O$ e $P$ da figura 1, respectivamente, a prova do teorema consiste então em escrever $m, n, o$ e $p$ em função de $z_{1}, z_{2}, z_{3}$ e $z_{4}$ e mostrar que $n-p=(m-o) i$, onde $i$ é o número complexo imaginário puro $i=\sqrt{-1}$. A identidade $n-p=(m-o) i$, uma vez demonstrada, revela, enfim, que os segmentos $O M$ e $P N$ são ortogonais e congruentes. Eis as ideias gerais da prova do teorema de Van Aubel que serão utilizadas, com detalhes, nas demonstrações do presente artigo para os octógonos.

Antes de iniciarmos a apresentação do resultado principal deste artigo, convém relembrarmos alguns fatos importantes relacionados aos números complexos e suas propriedades. Em particular, a interpretação geométrica do produto entre dois números complexos, que será mostrada a seguir. 
Quando um número complexo $z$, de módulo $r_{1}$ e argumento $\theta_{1}$ (em graus), é multiplicado por outro número complexo $w$, de módulo $r_{2}$ e argumento $\theta_{2}$, então o número complexo resultante $z \cdot w$ será aquele de módulo $r_{1} \cdot r_{2}$ e argumento $\theta_{1}+\theta_{2}$. Em outras palavras, denotando $z=x+y i$, então, ao $z$ ser multiplicado por $w$, o vetor de origem $(0,0) \mathrm{e}$ extremidade $(x, y)$ sofre uma rotação de $\theta_{2}$ graus em torno da origem, e o seu módulo fica multiplicado por $r_{2}$, sendo que, se $\theta_{2}$ for negativo, a rotação é no sentido horário, se positivo, no anti-horário.

Em particular, ao multiplicamos um número complexo $z$ pelo número imaginário puro $i$, então o complexo resultante será tal que seu módulo é igual ao de $z$, pois $|i|=1$, e tal que o seu argumento é o argumento de $z$ acrescido de $90^{\circ}$, pois o argumento de $i$ é $90^{\circ}$. Em outras palavras, denotando $z=x+i y$, o vetor de origem $(0,0)$ e extremidade $(x, y)$ é rotacionado em 90 graus no sentido anti-horário, e seu módulo permanece inalterado.

Cabem ainda mais alguns lembretes que serão usados. Dado o número complexo $z=x+$ $y i$, o ponto do plano $(x, y)$ é denominado de afixo de $z$. Dados dois números complexos $z_{1}=$ $x_{1}+i y_{1}$ e $z_{2}=x_{2}+i y_{2}$, então o ponto médio do segmento de extremidades $\left(x_{1}, y_{1}\right)$ e $\left(x_{2}, y_{2}\right)$ é dado pelo ponto $\left(\frac{x_{1}+x_{2}}{2}, \frac{y_{1}+y_{2}}{2}\right)$, que é o afixo do número complexo $\frac{z_{1}+z_{2}}{2}$.

Podemos, tendo feitas estas observações, iniciar a apresentação do resultado central deste artigo.

\section{Explorando o octógono}

Antes de enunciarmos e demonstrarmos o resultado deste artigo, é necessário que façamos a seguinte construção: dado um octógono $D_{1} D_{2} \ldots D_{8}$ qualquer, liguemos seus vértices opostos. Tomando os pontos médios $I, J, K$ e $L$ de tais segmentos, observamos que $\mathrm{o}$ quadrilátero $I J K L$, quando não degenerado em um ponto ou em um segmento, não forma necessariamente um quadrado ou quadrilátero notável, como paralelogramo ou retângulo, como ilustra a figura 2 .

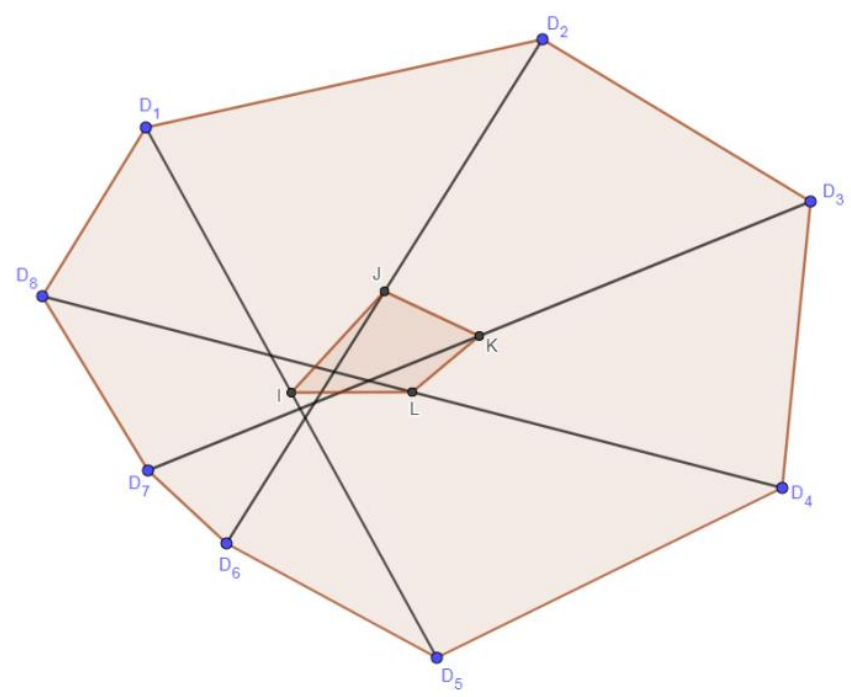

Figura 2 - Se $I, J, K, L$ não coincidem, eles formam um quadrilátero que não necessariamente é notável. Fonte: produção dos autores no software GeoGebra.

Queremos descobrir se há casos em que $I J K L$, quando não degenerado, é um quadrilátero notável. Então, vamos agora impor que $D_{1} D_{2} \ldots D_{8}$ não seja um octógono qualquer, mas que seja um octógono cujos vértices $D_{1}, \ldots, D_{8}$ são pontos médios dos lados de outro octógono, arbitrário, $C_{1} C_{2} \ldots C_{8}$. 
Nesta situação, o que dizer do quadrilátero $I J K L$, cujos vértices são, novamente, os pontos médios dos segmentos que unem os vértices opostos de $D_{1} D_{2} \ldots D_{8}$ ? Aqui, já temos algum resultado interessante: IJKL é um paralelogramo, como demonstra o trabalho de Santos, Comby e Silva (2019), e conforme ilustra a figura 3.

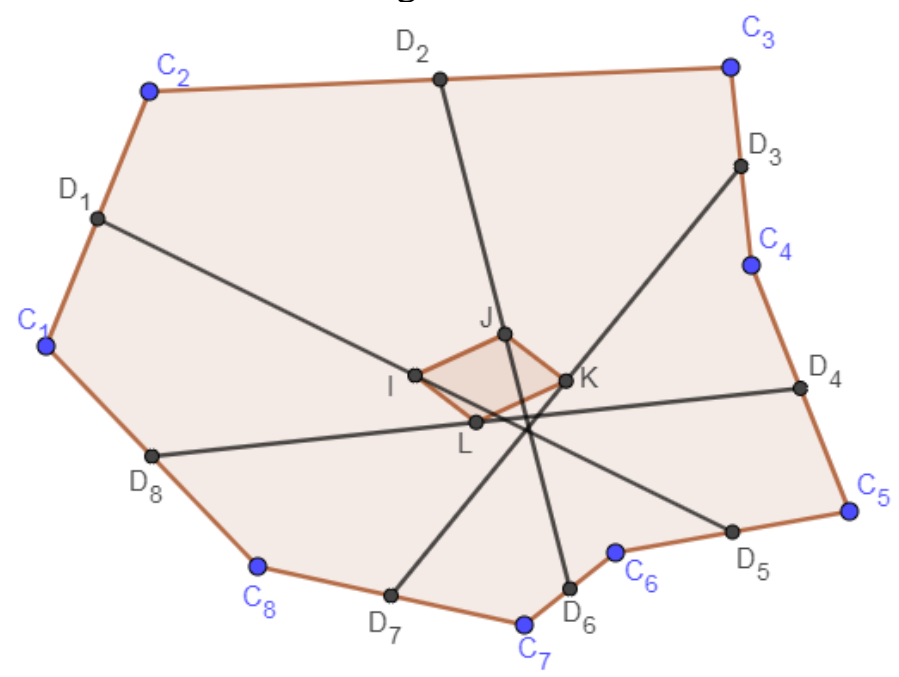

Figura 3 - Os pontos médios $I, J, K$ e $L$ dos segmentos cujos extremos são os pontos médios dos lados opostos de um octógono qualquer formam um paralelogramo.

Fonte: Santos, Comby e Silva (2019)

Logo, se impormos que $D_{1}, D_{2}, \ldots, D_{8}$ sejam pontos médios dos lados de um octógono arbitrário $C_{1} C_{2} \ldots C_{8}$, então $I J K L$ é um paralelogramo. A pergunta natural que podemos nos fazer ainda é: há ainda alguma restrição que possamos fazer, porém agora sobre o octógono $C_{1} C_{2} \ldots C_{8}$ da figura 3 , para o qual o quadrilátero central $I J K L$ seja um quadrado, quando não degenerado num ponto? A resposta é sim: basta que $C_{1}, C_{2}, \ldots, C_{8}$ sejam os centros dos quadrados construídos sobre algum outro octógono $A_{1} A_{2} \ldots A_{8}$ qualquer. É o que veremos no próximo capítulo, no qual provaremos o resultado principal deste artigo.

\section{Quadrados centrais associados a um octógono}

Considere um octógono qualquer $A_{1} A_{2} \ldots A_{8}$, não necessariamente convexo. Tome quadrados externos aos seus lados, cujos vértices externos são $B_{1}, B_{2}, \ldots, B_{16}$ (figura 4), sendo $C_{1}, C_{2}, \ldots, C_{8}$ os centros de tais quadrados. Tome $D_{1}, D_{2}, \ldots, D_{8}$ os pontos médios dos segmentos $C_{1} C_{2}, C_{2} C_{3}, \ldots, C_{8} C_{1}$, respectivamente.

Vamos provar neste artigo que, se $I, J, K$ e $L$ são os centros dos segmentos $D_{1} D_{5}, D_{2} D_{6}, D_{3} D_{7}$ e $D_{4} D_{8}$, respectivamente, então o quadrilátero $I J K L$ é um quadrado, chamado aqui de quadrado central associado ao octógono, como ilustra a figura 4. Observe que estamos na mesma construção feita no capítulo 2, ilustrada na figura 3, com uma suposição a mais: que $C_{1}, \ldots, C_{8}$ são centros de quadrados que jazem sobre os lados de um octógono qualquer $A_{1} A_{2} \ldots A_{8}$. 


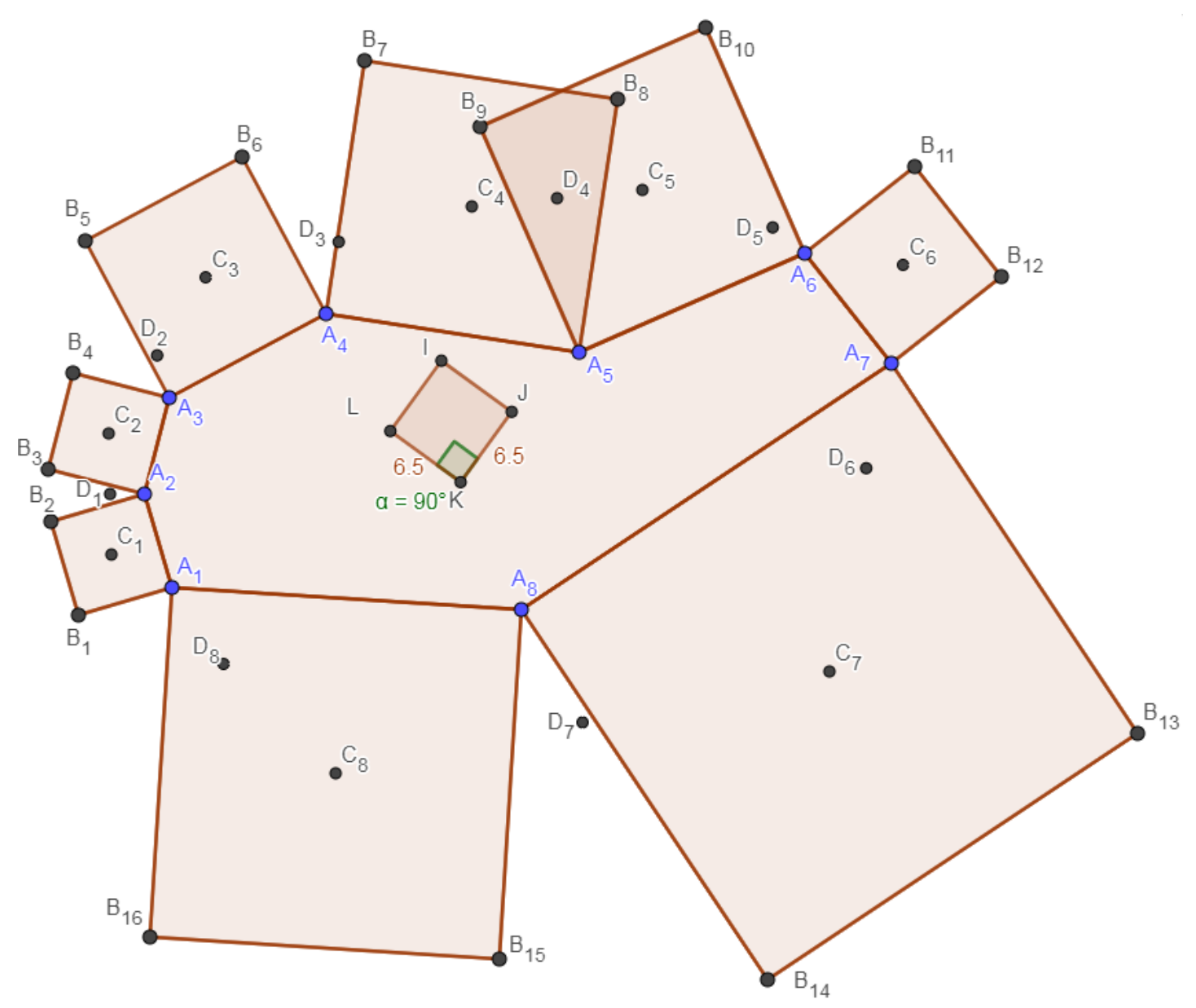

Figura 4 - IJKL é um quadrado quando $C_{1}, C_{2}, \ldots$ e $C_{8}$ são centros de quadrados construídos externamente sobre os lados de um octógono qualquer $A_{1} A_{2} \ldots A_{8}$. Fonte: retirado de Silva (2018).

Além de mostrar que $I J K L$ é um quadrado neste caso, vamos provar também que, no mesmo octógono $A_{1} A_{2} \ldots A_{8}$, se, ao invés de tomarmos os quadrados externos aos seus lados, tomarmos os oito quadrados internos sobre os lados do mesmo, o respectivo quadrilátero $I^{\prime} J^{\prime} K^{\prime} L^{\prime}$, obtido da mesma forma que o anterior, também será um quadrado, e mais, os centros dos dois quadrados centrais associados ao octógono $I J K L$ e $I^{\prime} J^{\prime} K^{\prime} L^{\prime}$ coincidem.

A figura 5 ilustra o fato de que $I^{\prime} J^{\prime} K^{\prime} L^{\prime}$ também é um quadrado, e ainda, que os centros dos dois quadrados $I J K L$ e $I^{\prime} J^{\prime} K^{\prime} L^{\prime}$ coincidem. Omitimos na figura os quadrados construídos internamente sobre os lados do octógono para não poluir a visualização da mesma. No entanto, estão indicados os centros $C^{\prime}{ }_{j}, j=1, \ldots, 8$ dos quadrados construídos internamente sobre os lados do octógono, bem como os pontos médios $D^{\prime}{ }_{j}, j=1, \ldots, 8$ de cada segmento $C^{\prime}{ }_{j} C_{j+1}^{\prime}$, em que $I^{\prime}, J^{\prime}, K^{\prime}$ e $L^{\prime}$ são os pontos médios de $D^{\prime}{ }_{1} D^{\prime}{ }_{5}, D^{\prime}{ }_{2} D_{6}^{\prime}{ }_{6}, D^{\prime}{ }_{3} D^{\prime}{ }_{7}$ e $D^{\prime}{ }_{4} D^{\prime}{ }_{8}$, respectivamente. Estamos considerando que $C_{9}^{\prime}=C_{1}^{\prime}$. 


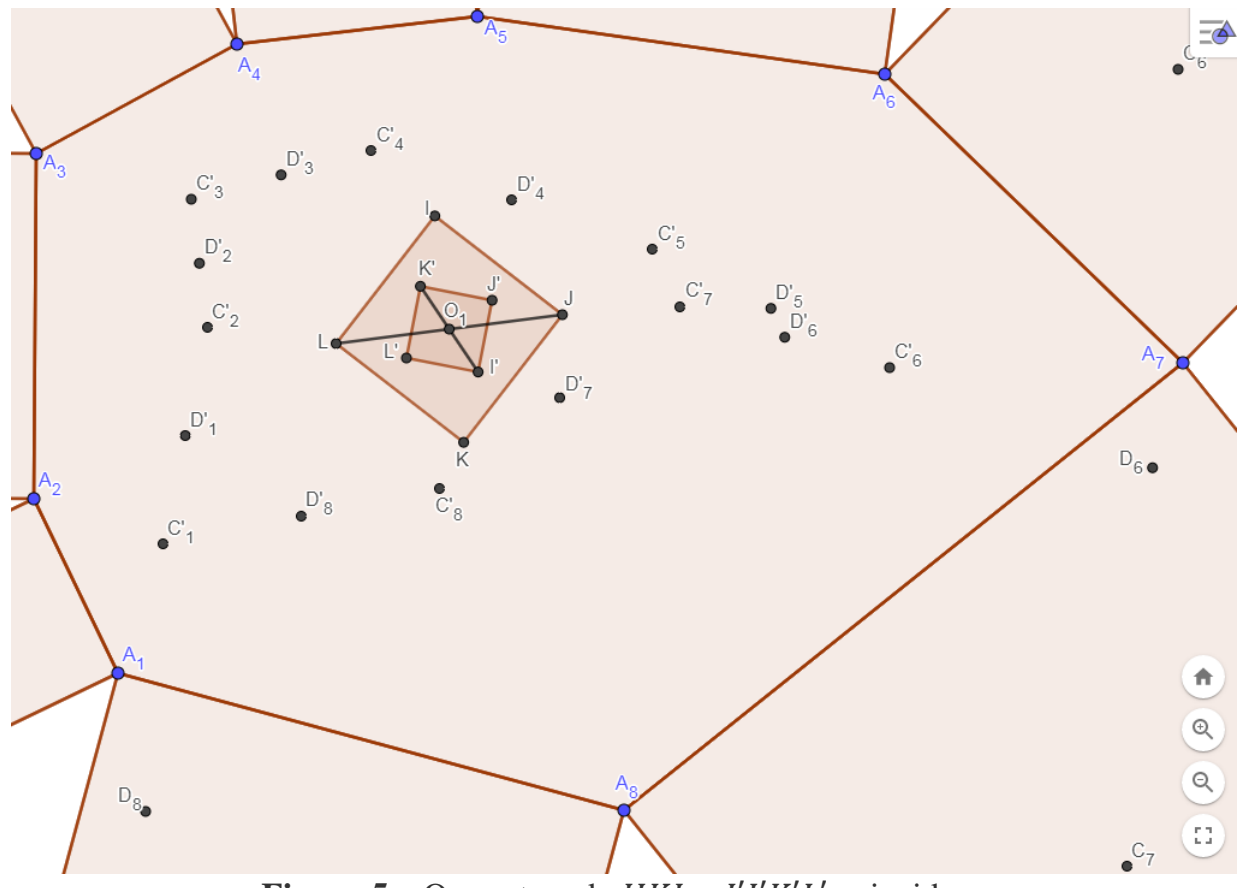

Figura 5 - Os centros de $I J K L$ e $I^{\prime} J^{\prime} K^{\prime} L^{\prime}$ coincidem Fonte: produção dos autores no software GeoGebra.

Antes das provas, cabem duas observações. Uma observação é que, se $I, J, K$ e $L$ fossem diretamente os centros dos segmentos $C_{j} C_{j+4}$, ou seja, se não tomamos os $D_{j}$, então IJKL não seria um quadrado ou outra figura notável, conforme mostra a figura 6:

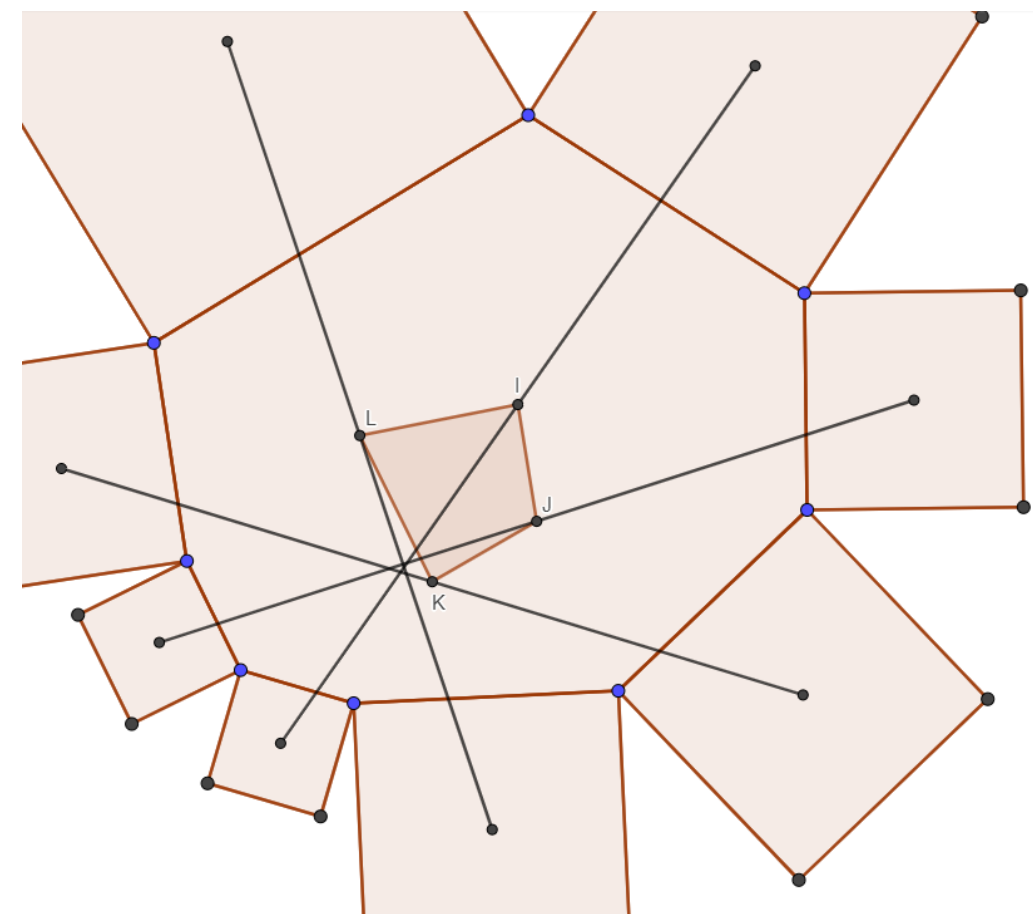

Figura 6 - Se $I, J, K$ e $L$ são os pontos médios dos segmentos que unem diretamente os centros opostos dos oito quadrados, então $I J K L$ não necessariamente é um quadrilátero notável.

Fonte: produção dos autores no software GeoGebra.

Outra observação, agora sobre o trabalho de Silva (2018). Lá, o autor demonstra que, se continuássemos a construção pegando agora os pontos médios $E_{1}, \ldots, E_{8}$, dos segmentos 
consecutivos $D_{1} D_{2}, \ldots, D_{8} D_{1}$, então os quatro pontos médios dos quatro segmentos $E_{1} E_{5}, E_{2} E_{6}, E_{3} E_{7}$ e $E_{4} E_{8}$, que podemos denotar por $I_{E}, J_{E}, K_{E}$ e $L_{E}$, formam um quadrado. Afigura 7 ilustra.

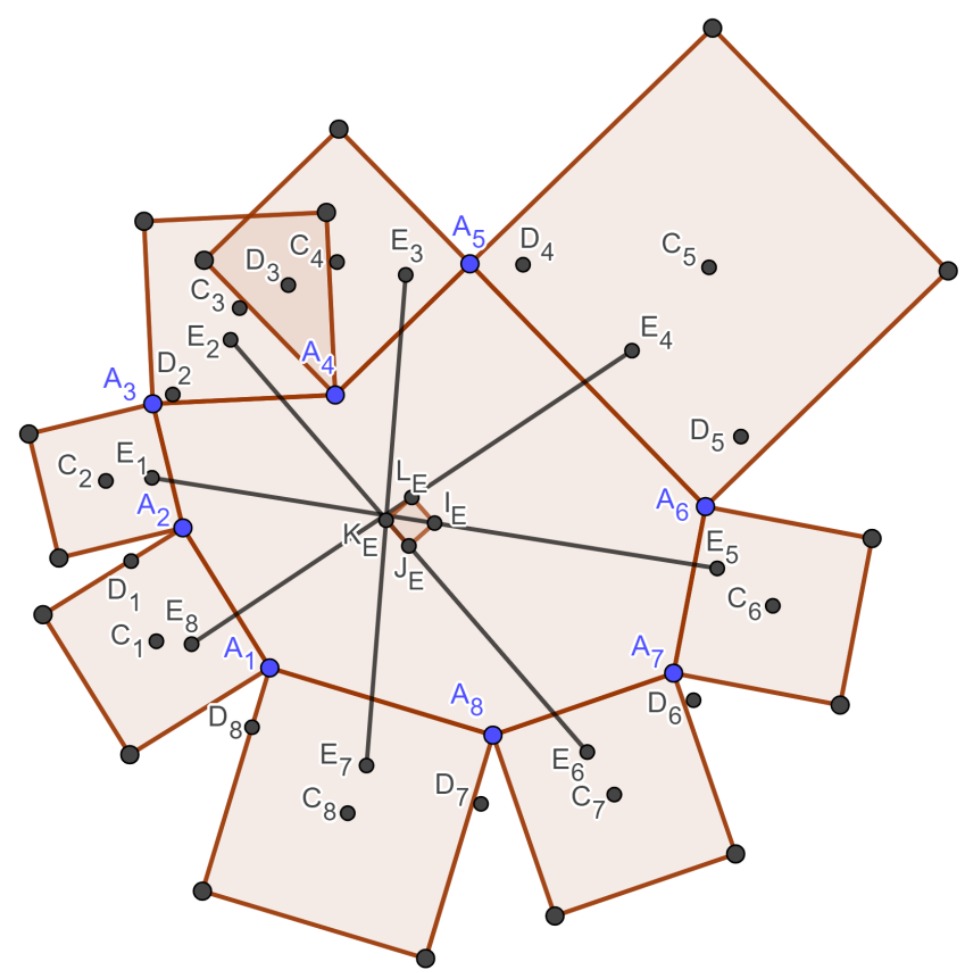

Figura $7-I_{E} J_{E} K_{E} L_{E}$ é um quadrado também, quando construído a partir dos pontos médios de $E_{j} E_{j+4}$. Fonte: produção dos autores no software GeoGebra.

Nesta construção de Silva (2018), em que consideramos os pontos $E_{j}$, médios de $D_{i} D_{j+1}$, é possível provar também que, se os quadrados forem construídos internamente ao octógono $A_{1} A_{2} \ldots A_{8} A_{1}$, então $I_{E}^{\prime} J_{E}^{\prime} K_{E}^{\prime} L_{E}^{\prime}$ também se constitui num quadrado, e os centros dos dois quadrados coincidem.

Feita essa observação relativa ao trabalho de Silva (2018), vamos demonstrar então nossa proposição de que $I J K L$ e $I^{\prime} J^{\prime} K^{\prime} L^{\prime}$ são quadrados e que possuem o mesmo centro, em que pegamos somente até os pontos $D_{j}\left(D_{j}^{\prime}\right.$, respectivamente), conforme ilustram as figuras 4 e 5 .

\section{Demonstração.}

A figura 4 está reproduzida na figura 8 para um melhor acompanhamento. Considere que os pontos da figura 8 são tomados como sendo os afixos de seus respectivos números complexos. Do modo como foi feita a construção, inserindo quadrados sobre os lados do polígono, nota-se a familiaridade com o teorema de Van Aubel. A prova segue os mesmo princípios. 


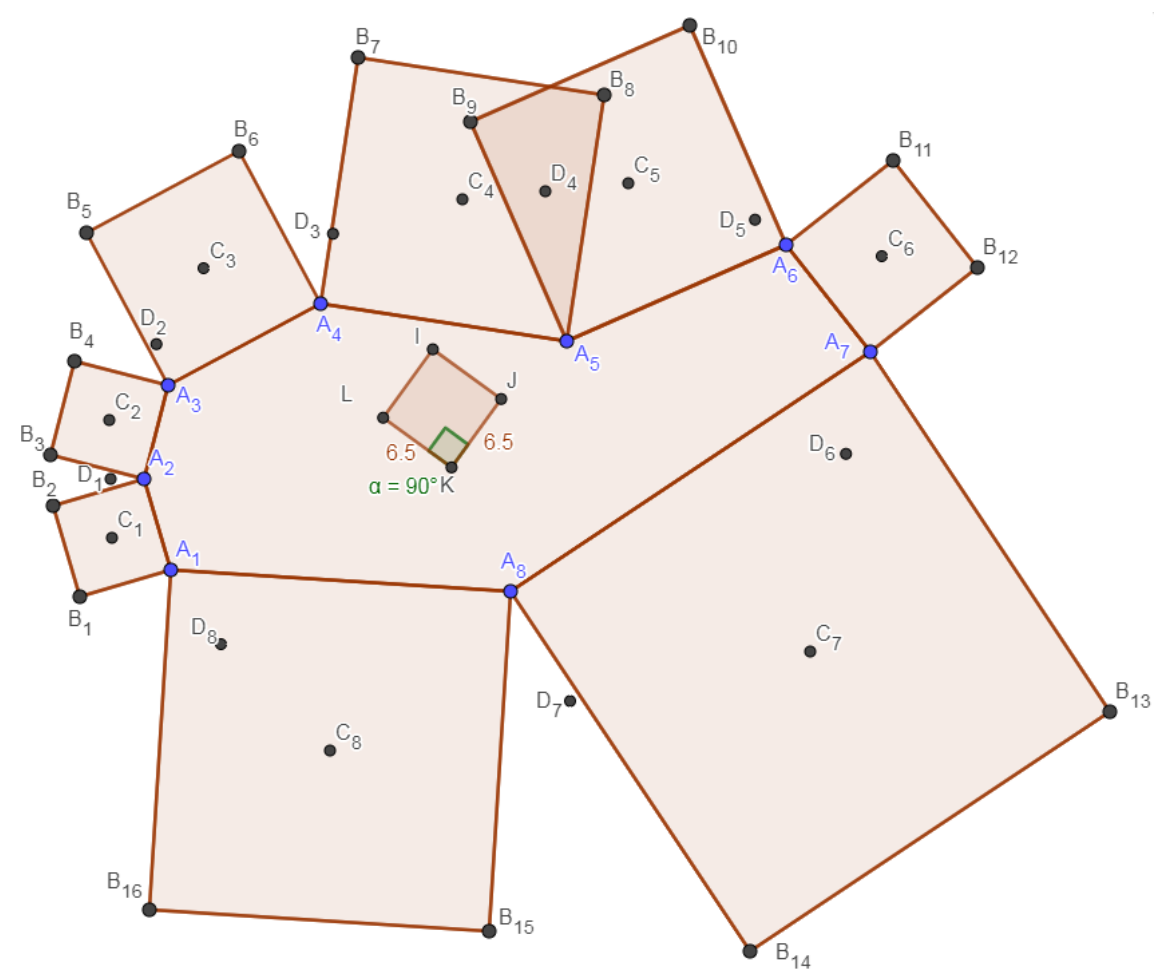

Figura 8 - Redução da figura 4 para acompanhamento da prova. Fonte: produção dos autores no software GeoGebra.

Sejam $a_{1}, a_{2}, \ldots, a_{8}$ os números complexos correspondentes aos pontos afixos $A_{1}, A_{2}, \ldots, A_{8}$, respectivamente, isto é, se $A_{1}=(y, z)$, então $a_{1}=y+i z$, por exemplo.

Defina ainda, como em Silva (2018), $2 a, 2 b, 2 c, 2 d, 2 e, 2 f, 2 g$ e $2 h \in \mathbb{C}$ os números complexos correspondentes aos pontos afixos: $A_{2}-A_{1}, A_{3}-A_{2}, A_{4}-A_{3}, \ldots, A_{1}-A_{8}$, respectivamente, isto é, $2 a, 2 b, 2 c, 2 d, 2 e, 2 f, 2 g$ e $2 h$ são os números complexos correspondentes aos vetores $\overrightarrow{A_{1} A_{2}}, \overrightarrow{A_{2} A_{3}}, \ldots, \overrightarrow{A_{8} A_{1}}$, os lados do octógono.

Por ser uma figura fechada, temos que

$$
\begin{gathered}
2 a+2 b+2 c+2 d+2 e+2 f+2 g+2 h=0 \therefore \\
a+b+c+d+e+f+g+h=0 .
\end{gathered}
$$

Denotando temporariamente por $M=\frac{A_{1}+A_{2}}{2}$ o ponto médio do segmento $A_{1} A_{2}$, por $m$ o número complexo associado ao afixo $M$, e por $c_{j}$ os números complexos associados aos afixos $C_{j}, j=1, \ldots, 8$, temos, pela figura 9 :

$$
c_{1}-m=\left(m-a_{1}\right) i
$$

pois, a rotação de $90^{\circ}$ no sentido anti-horário do vetor $\overrightarrow{A_{1} M}$ resulta em um vetor equivalente ao vetor $\overrightarrow{M C_{1}}$ (figura 9), a menos de uma translação no plano. 


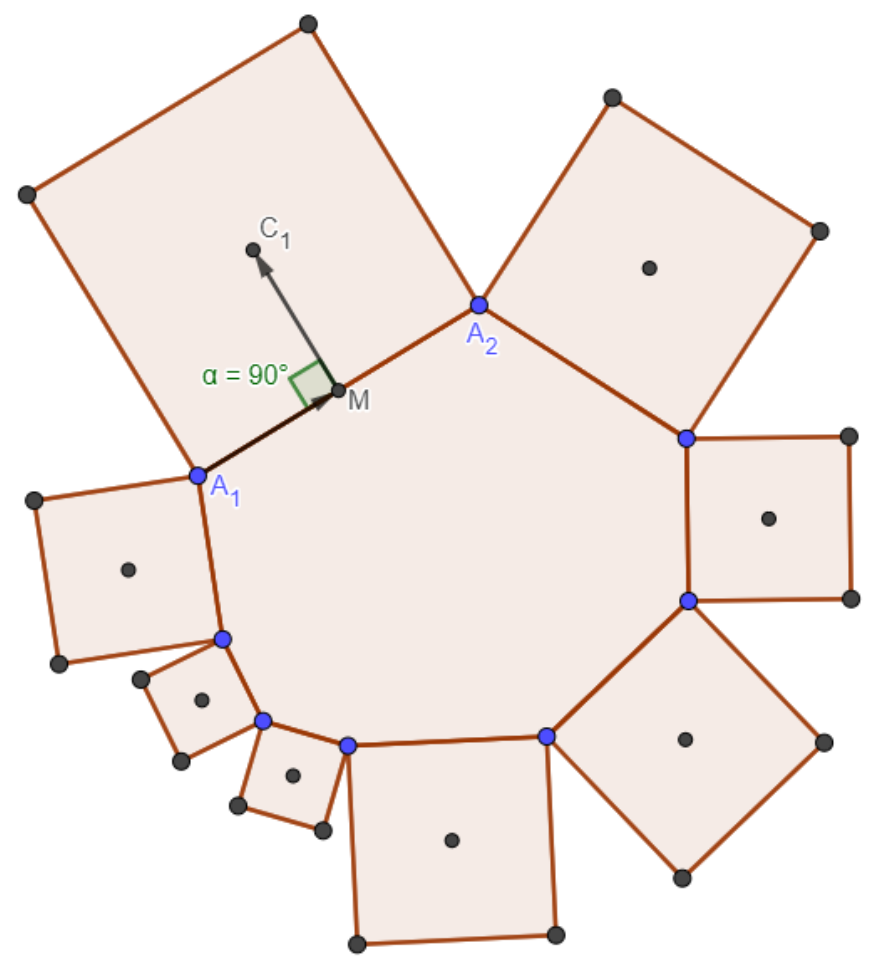

Figura 9 - A rotação do vetor $\overrightarrow{A_{1} M}$ em $90^{\circ}$ no sentido anti-horário resulta no vetor $\overrightarrow{M C_{1}}$, a menos de uma translação.

Fonte: construído no software GeoGebra

Sem perda de generalidade, podemos considerar que $A_{1}$ é o a origem $(0,0)$ do plano cartesiano, isto é, $a_{1}=0+0 i=0$. Então, como tínhamos que $2 a$ era o número complexo associado ao ponto afixo $A_{2}-A_{1}$, mas sabendo que

$$
A_{2}-A_{1}=A_{2}-(0,0)=A_{2} \text {, }
$$

então $A_{2}$ é o ponto afixo do número complexo $2 a$ e, portanto, $m=\frac{a_{1}+a_{2}}{2}=\frac{0+2 a}{2}=a$. Assim:

$$
\begin{gathered}
c_{1}-m=\left(m-a_{1}\right) i, \\
c_{1}-a=(a-0) i, \\
c_{1}=a+a i=a(1+i) .
\end{gathered}
$$

Apropriando-nos da letra $M$ para denotar agora o ponto médio de $A_{2} A_{3}$, temos, pela figura 10 , que $\overrightarrow{A_{1} A_{2}}+\overrightarrow{A_{2} M}+\overrightarrow{M C_{2}}=\overrightarrow{A_{1} C_{2}}$, isto é,

$$
\left(A_{2}-A_{1}\right)+\left(M-A_{2}\right)+\left(C_{2}-M\right)=C_{2}-A_{1} .
$$

Lembremo-nos por um instante de alguns fatos relativos aos termos dentro dos parênteses:

- ao afixo $A_{2}-A_{1}$ corresponde o número complexo $2 a$,

- ao afixo $M-A_{2}$ corresponde o número complexo $b$, pois $2 b$ corresponde ao afixo $A_{3}-A_{2}$; e isto implica em dizer que $m-a_{2}=b$,

- ao afixo $C_{2}-M$ corresponde o número complexo $\left(m-a_{2}\right) i=b i$, pois o vetor $\overrightarrow{M C_{2}}$ é equivalente ao vetor resultante da rotação de $\overrightarrow{A_{2} M}$ em $90^{\circ}$ no sentido anti-horário (figura 7), e

Logo, a equação

- ao afixo $C_{2}-A_{1}$ corresponde o número complexo $c_{2}$, pois $A_{1}=(0,0)$.

implica na equação

$$
\left(A_{2}-A_{1}\right)+\left(M-A_{2}\right)+\left(C_{2}-M\right)=C_{2}-A_{1}
$$

$$
2 a+b+b i=c_{2} .
$$


Logo,

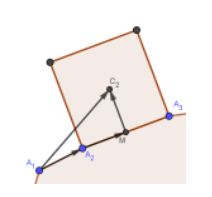

Figura 10 - Soma de vetores $\overrightarrow{A_{1} A_{2}}+\overrightarrow{A_{2} M}+\overrightarrow{M C_{2}}=\overrightarrow{A_{1} C_{2}}$

Fonte: construído no software GeoGebra.

$$
c_{2}=2 a+b(1+i)
$$

Por fim, pensando de forma análoga, obtemos todos os números complexos $c_{j}$ correspondentes aos centros $C_{j}$ dos quadrados, em termos dos lados do octógono:

$$
\begin{gathered}
c_{1}=a(1+i), \\
c_{2}=2 a+b(1+i), \\
c_{3}=2 a+2 b+c(1+i), \\
c_{4}=2 a+2 b+2 c+d(1+i), \\
c_{5}=2 a+2 b+2 c+2 d+e(1+i), \\
c_{6}=2 a+2 b+2 c+2 d+2 e+f(1+i), \\
c_{7}=2 a+2 b+2 c+2 d+2 e+2 f+g(1+i), \\
c_{8}=2 a+2 b+2 c+2 d+2 e+2 f+2 g+h(1+i) .
\end{gathered}
$$

Lembrando que $D_{1}, D_{2}, \ldots, D_{8}$ são os pontos médios de $C_{1} C_{2}, C_{2} C_{3}, \ldots, C_{8} C_{1}$, respectivamente, podemos obter, em termos de números complexos:

$$
\begin{gathered}
d_{1}=\frac{c_{1}+c_{2}}{2}=\frac{3 a+b+(a+b) i}{2}, \\
d_{2}=\frac{c_{2}+c_{3}}{2}=\frac{4 a+3 b+c+(b+c) i}{2}, \\
d_{3}=\frac{c_{3}+c_{4}}{2}=\frac{4 a+4 b+3 c+d+(c+d) i}{2}, \\
d_{4}=\frac{c_{4}+c_{5}}{2}=\frac{4 a+4 b+4 c+3 d+e+(d+e) i}{2}, \\
d_{5}=\frac{c_{5}+c_{6}}{2}=\frac{4 a+4 b+4 c+4 d+3 e+f+(e+f) i}{2}, \\
d_{6}=\frac{c_{6}+c_{7}}{2}=\frac{4 a+4 b+4 c+4 d+4 e+3 f+g+(f+g) i}{2}, \\
d_{7}=\frac{c_{7}+c_{8}}{2}=\frac{4 a+4 b+4 c+4 d+4 e+4 f+3 g+h+(g+h) i}{2} \mathrm{e}
\end{gathered}
$$




$$
d_{8}=\frac{c_{8}+c_{1}}{2}=\frac{3 a+2 b+2 c+2 d+2 e+2 f+2 g+h+(a+h) i}{2} .
$$

Além disso, $I, J, K$ e $L$ são os centros de $D_{1} D_{5}, D_{2} D_{6}, D_{3} D_{7}$ e $D_{4} D_{8}$, respectivamente, assim, se usarmos as mesmas notações dos pontos $I, J, K$ e $L$ para os números complexos respectivos aos seus afixos, temos:

$$
\begin{aligned}
& I=\frac{d_{1}+d_{5}}{2}= \\
& \frac{1}{2} \cdot\left(\frac{3 a+b+(a+b) i}{2}+\frac{4 a+4 b+4 c+4 d+3 e+f+(e+f) i}{2}\right)= \\
& \frac{7 a+5 b+4 c+4 d+3 e+f+(a+b+e+f) i}{4} \\
& J=\frac{d_{2}+d_{6}}{2}= \\
& \frac{1}{2} \cdot\left(\frac{4 a+3 b+c+(b+c) i+4 a+4 b+4 c+4 d+4 e+3 f+g+(f+g) i}{2}\right)= \\
& \frac{8 a+7 b+5 c+4 d+4 e+3 f+g+(b+c+f+g) i}{4} \\
& K=\frac{d_{3}+d_{7}}{2}= \\
& \frac{1}{2} \cdot\left(\frac{4 a+4 b+3 c+d+(c+d) i+4 a+4 b+4 c+4 d+4 e+4 f+3 g+h+(g+h) i}{2}\right) \\
& = \\
& \frac{8 a+8 b+7 c+5 d+4 e+4 f+3 g+h+(c+d+g+h) i}{4} \mathrm{e} \\
& L=\frac{d_{4}+d_{8}}{2}= \\
& \frac{4 a+4 b+4 c+3 d+e+(d+e) i+3 a+2 b+2 c+2 d+2 e+2 f+2 g+h+(a+h) i}{4} \\
& = \\
& \frac{7 a+6 b+6 c+5 d+3 e+2 f+2 g+h+(a+d+e+h) i}{4} .
\end{aligned}
$$

Substituindo estes valores na equação $(J-I)-i(L-I)=0$, esta igualdade se verifica, conforme será mostrado a seguir, o que implica que os segmentos adjacentes $I J$ e $I L$ do quadrilátero $I J K L$ são ortogonais e congruentes, vejamos:

$$
\begin{gathered}
J-I= \\
\frac{8 a+7 b+5 c+4 d+4 e+3 f+g+(b+c+f+g) i}{4} \\
-\frac{7 a+5 b+4 c+4 d+3 e+f+(a+b+e+f) i}{4}=
\end{gathered}
$$




$$
\frac{a+2 b+c+e+2 f+g+(-a+c-e+g) i}{4} .
$$

Por outro lado,

$$
\begin{gathered}
L-I= \\
\frac{7 a+6 b+6 c+5 d+3 e+2 f+2 g+h+(a+d+e+h) i}{4} \\
-\frac{7 a+5 b+4 c+4 d+3 e+f+(a+b+e+f) i}{4}= \\
\frac{b+2 c+d+f+2 g+h+(-b+d-f+h) i}{4} .
\end{gathered}
$$

Agora,

$$
\begin{gathered}
J-I-i(L-I)= \\
=\frac{a+2 b+c+e+2 f+g+(-a+c-e+g) i}{4} \\
-i \cdot\left(\frac{b+2 c+d+f+2 g+h+(-b+d-f+h) i}{4}\right)= \\
+\frac{-(b+2 c+d+f+2 g+h) i-b+d-f+h}{4}= \\
\frac{a+b+c+d+e+f+g+h+(-a-b-c-d-e-f-g-h) i}{4}=0+0 i=0 .
\end{gathered}
$$

Analogamente se mostra para os demais pares lados adjacentes do quadrilátero $I J K L$. Portanto IJKL é um quadrado.

Consideremos agora os quadrados construídos internamente ao octógono, com as mesmas notações anteriores, sem perigo de confusão, isto é, $M$ é o ponto médio do segmento $A_{1} A_{2}$, $a_{1}=0, a_{2}=2 a$ e $m=\frac{a_{1}+a_{2}}{2}=a$.

Ora, como $C_{1}$ agora é o centro do quadrado interno ao lado do octógono, temos, portanto:

$$
c_{1}-m=\left(m-a_{1}\right)(-i) \text {, }
$$

pois a rotação de $90^{\circ}$ no sentido horário do vetor $\overrightarrow{A_{1} M}$ resulta em um vetor equivalente ao vetor $\overrightarrow{M C_{1}}$.

Daí,

De maneira análoga, temos:

$$
\begin{aligned}
& c_{1}-a=-a i, \\
& c_{1}=a(1-i) .
\end{aligned}
$$

$$
\begin{gathered}
c_{2}=2 a+b(1-i), \\
\vdots \\
c_{8}=2 a+2 b+2 c+2 d+2 e+2 f+2 g+h(1-i) .
\end{gathered}
$$


A demonstração é análoga ao caso dos quadrados construídos externamente ao octógono, por isso omitiremos desta vez os detalhes dos passos.

Continuando, temos:

$$
\begin{gathered}
d_{1}=\frac{c_{1}+c_{2}}{2}=\frac{3 a+b-(a+b) i}{2}, \\
d_{8}=\frac{c_{8}+c_{1}}{2}=\frac{3 a+2 b+2 c+2 d+2 e+2 f+2+h-(a+h) i}{2} .
\end{gathered}
$$

Observe que os $d_{j}$ não são iguais aos conjugados complexos dos $d_{j}$ anteriores, pois $a, b, \ldots, h$ são números complexos, porém a única diferença de um para o outro é o sinal negativo antes do termo que multiplica $i$.

Tomando então $I^{\prime}, J^{\prime}, K^{\prime}$ e $L^{\prime}$ os centros de $D_{1} D_{5}, D_{2} D_{6}, D_{3} D_{7}$ e $D_{4} D_{8}$, respectivamente, e calculando $I^{\prime}=\frac{d_{1}+d_{5}}{2}, J^{\prime}=\frac{d_{2}+d_{6}}{2}, K^{\prime}=\frac{d_{3}+d_{7}}{2}$ e $L^{\prime}=\frac{d_{4}+d_{8}}{2}$, a demonstração de que $J^{\prime}-I^{\prime}-$ $i\left(I^{\prime}-L^{\prime}\right)=0$ torna-se inteiramente análoga ao caso anterior dos quadrados externos.

Assim, os lados consecutivos $I^{\prime} J^{\prime}$ e $I^{\prime} L^{\prime}$ são congruentes e ortogonais. Podemos fazer o mesmo para os demais lados adjacentes do quadrilátero $I^{\prime} J^{\prime} K^{\prime} L^{\prime}$. Portanto, $I^{\prime} J^{\prime} K^{\prime} L^{\prime}$ é um quadrado.

Consideremos, portanto, os quadrados $I J K L$ e $I^{\prime} J^{\prime} K^{\prime} L^{\prime}$. Vamos mostrar que eles possuem o mesmo centro, objetivo final deste artigo. Para tanto, iremos considerar as diagonais $I K$ e $I^{\prime} K^{\prime}$ dos dois quadrados e calcular os seus pontos médios.

Considerando o quadrado $I J K L$, temos que o ponto médio $O_{1}$ da diagonal $I K$ pode ser escrito como:

$$
\begin{gathered}
O_{1}=\frac{I+K}{2}= \\
\frac{7 a+5 b+4 c+4 d+3 e+f+(a+b+e+f) i}{8}+ \\
\frac{8 a+8 b+7 c+5 d+4 e+4 f+3 g+h+(c+d+g+h) i}{8}=
\end{gathered}
$$

ou seja,

$$
\frac{\begin{array}{c}
O_{1}= \\
15 a+13 b+11 c+9 d+7 e+5 f+3 g+h+(a+b+c+d+e+f+g+h) i \\
\frac{8}{8} a+13 b+11 c+9 d+7 e+5 f+3 g+h \\
8
\end{array}}{0 .}
$$

pois $a+b+c+d+e+f+g+h=0$.

Considerando o quadrado $I^{\prime} J^{\prime} K^{\prime} L^{\prime}$, e considerando que a diferença na representação em relação ao $I J K L$ está apenas no sinal antes de $i$, temos que o ponto médio $O_{2}$ da diagonal $I^{\prime} K^{\prime}$ pode ser escrito como:

$$
\begin{gathered}
O_{2}=\frac{I^{\prime}+K^{\prime}}{2}= \\
\frac{15 a+13 b+11 c+9 d+7 e+5 f+3 g+h-(a+b+c+d+e+f+g+h) i}{8}= \\
\frac{15 a+13 b+11 c+9 d+7 e+5 f+3 g+h}{8} .
\end{gathered}
$$

Portanto, temos que $O_{1}=O_{2}$, ou seja, os quadrados $I J K L$ e $I^{\prime} J^{\prime} K^{\prime} L^{\prime}$ possuem o mesmo centro, como queríamos demonstrar. 
É possível mostrar ainda (os cálculos são semelhantes aos feitos acima, desnecessários neste momento) que o quadrado $I J K L$ aqui considerado e ilustrado na figura 8 possui a seguinte relação com o quadrado correspondente $I_{E} J_{E} K_{E} L_{E}$ quando são considerados os pontos $E_{j}$ do trabalho de Silva (2018): os vértices do quadrado central $I_{E} J_{E} K_{E} L_{E}$ de Silva (2018) são pontos médios dos lados do nosso quadrado central $I J K L$ (sem considerar os $E_{j}$ ). A figura 11 ilustra, sem muitos elementos para não poluir a figura.

Ainda mais, aumentando a construção e considerando ainda pontos $F_{j}$, pontos médios dos segmentos $E_{j} E_{j+4}$, o quadrado correspondente $I_{F} J_{F} K_{F} L_{F}$ também terá vértices nos pontos médios do quadrado $I_{E} J_{E} K_{E} L_{E}$ anterior, e assim por diante. Assim, os centros de todos os infinitos quadrados coincidem, um sempre estará com a diagonal rotacionada em relação à diagonal do quadrado anterior, e os comprimentos dos lados dos quadrados tendem a zero (figura 11).

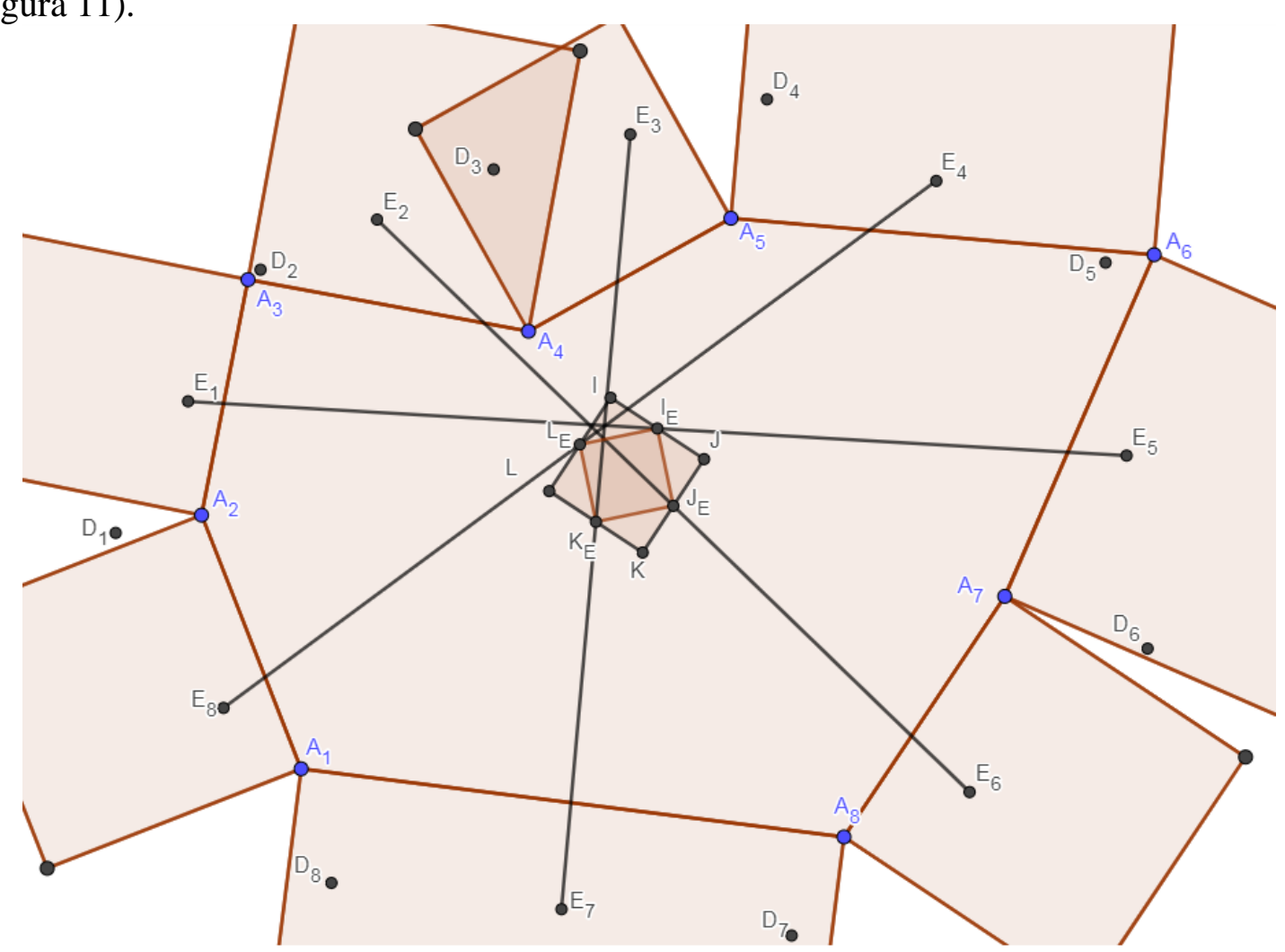

Figura 11 - Relação entre $I J K L$ e $I_{E} J_{E} K_{E} L_{E}$.

Fonte: construído no software GeoGebra.

\section{Conclusão}

Pudemos perceber que construções envolvendo pontos médios de segmentos ou centros de polígonos regulares podem acarretar em propriedades inéditas em Geometria Plana. Também verificamos que os números complexos podem ser usados para se obter rotação de vetores e, desta forma, mostram-se úteis às demonstrações em Geometria Euclidiana, como foi o caso das proposições mostradas aqui para os octógonos.

\section{Referências}

KRISHNA, D. N. V. An equilateral triangle associated with a hexagon. The Mathematical Gazette, v. 102, n. 555, p. 515-516, 2018. 
NISHIYAMA, Y. The beautiful geometric theorem of Van Aubel. International Journal of Pure and Applied Mathematics, v. 66, n. 1, p. 71-80, 2011.

SANTOS, R. C.; COMBY, A. C. O.; SILVA, B. R. V. Uma propriedade curiosa dos octógonos. Revista do Professor de Matemática. n. 99, p. 21-22, 2019.

SILVA, B. R. V. Teorema de Van Aubel: uma abordagem em sala de aula e demonstração de um caso similar. 2018. Dissertação. (Mestrado Profissional em Matemática) - Instituto de Ciências Exatas, Universidade de Brasília, Brasília, 2018. 